\title{
Dealing with Desire to Die in Patients with Incurable Cancer: Recommendations of the German Evidence-Based Guideline Palliative Care
}

\author{
Kremeike $K^{1}$, Pralong $A^{1}$, Simon $S T^{1}$, Bausewein $C^{2}$, Voltz $R^{1,3}$, Lindner $R^{4}$ \\ on behalf of the "working group desire to die" of the German Guideline Group Palliative Care \\ 1. University of Cologne, Faculty of Medicine and University Hospital, Department of Palliative Medicine \\ 2. Ludwig-Maximilians-University Munich, Munich University Hospital, Department of Palliative Medicine \\ 2. University of Cologne, Faculty of Medicine and University Hospital, Center for Integrated Oncology \\ 4. University Kassel, Faculty of Humanities, Institute of Social Work
}

\section{Background and Aims}

Desire to die (DD) is not uncommon in palliative patients. Although medical progres and improved therapy options can provide support and alleviate symptom burden, they do not prevent these wishes. Confronted with incurable cancer, many patients think about their future and meaning of life.

Health practitioners are often confronted with these thoughts or a desire to die. However, health practitioners often report uncertainty regarding background, meaning, functions and handling of DD.

This chapter of the Guideline Palliative Medicine presents the current national and international state of knowledge and experience in dealing with DD to offer orientation and assurance in responding to it in an appropriate manner.

\section{Methods}

Representatives from 60 professional societies developed and consented evidence and consensus based recommendations. Current evidence was identified by systematically searching the literature for systematic reviews and primary studies in Medline, Psyclnfo and the Cochrane Library.

Publications were selected according to previously defined inclusion criteria and assessed on their methodological quality according to the Scottish Intercollegiate Guidelines Network (SIGN).

The recommendations based on the identified evidence were approved through a formal agreement process at a consensus conference. Recommendations were graded from A (strong) to B (conditional) to 0 (open).

The presented results are part of the preliminary version of the S3 Guideline Palliative Medicine which is currently in the finalization phase and subject to modification. Near-term publication of its finalized version is scheduled.

\section{Results}

The expert panel agreed on 19 statements and recommendations dealing with DD issues such as differential diagnosis, potential background and meaning, how to deal with DD, possible options for action and the legal framework. A DD is understood as a complex phenomenon with individual differences in causes, manifestations and consequences. Sensitive communication about a DD is encouraged, either proactively initiated by the health professional or if mentioned by the patient. Evidence-based recommendations include, for example, the following:

18.28 Strength of recommendation B Level of Evidence 4

\subsection{Strength of recommendation A Level of Evidence 3}

\subsection{Evidence-based Statement Level of Evidence 3}

If a patient with incurable cancer expresses a desire to die, the existence of helplessness, hopelessness, meaninglessness, futility, demoralisation, loss of faith as well as depression and suicidal ideation should be assessed and treated or offered assistance for.

Patients with incurable cancer and a desire to die can simultaneously hold a wish to live. The desire to die and the wish to live can change over the course of time and regarding intensity.

The handling of DD within the multiprofessional treatment team, possible support from external experts and the involvement of relatives are also covered in the guideline.

\section{Discussion \& Conclusion}

The guideline aims to support decision-making in practice and provides systematically developed recommendations on the basis of the best possible evidence and clinical experience of a large number of experts. The recommendations concerning DD aim at helping with understanding its complexity and functions, developing an individual attitude towards it and gaining security in communicating about it. The recommendation to (proactively) address potential DD is a considerable novelty to the prior version of the S3 Guideline. Its addition illustrates the tremendous increase in literature over the last years concerning addressing and dealing with a DD. Therefore, the recommendations should be considered as a contribution to the advancement of multi-professional competence in palliative care.

Funding: German Guideline Program in Oncology (GGPO) 